\title{
NRSF/REST regulates the $m$ TOR signaling pathway in oral cancer cells
}

\author{
${\text { EUGENE } \mathrm{CHO}^{1 *}, \text { SUNG-MIN MOON }^{1,2^{*}}, \text { BO RAM PARK }}^{1,2}$, DO KYUNG KIM ${ }^{1}$, \\ BYUNG-KWON LEE ${ }^{3}$ and CHUN SUNG KIM ${ }^{1,2}$ \\ ${ }^{1}$ Oral Biology Research Institute, Chosun University; ${ }^{2}$ Department of Oral Biochemistry, \\ College of Dentistry, Chosun University, Dong-gu, Gwangju 501-759, Republic of Korea; \\ ${ }^{3}$ Department of Microbiology, University of Tennessee, Knoxville, TN 37996, USA
}

Received August 25, 2014; Accepted November 21, 2014

DOI: $10.3892 /$ or.2014.3675

\begin{abstract}
The neuron-restrictive silencer factor/repressor element 1-silencing transcription factor (NRSF/REST) was originally discovered as a transcriptional repressor of neuronal genes in non-neuronal cells. However, it was recently reported to be abundantly expressed in several types of aggressive cancer cells, as well as in mature neurons. In the present study, the role of NRSF/REST in the human oral squamous cell carcinoma (SCC) KB cell line was evaluated. NRSF/REST was expressed at a higher level in KB cells when compared with that in normal human oral keratinocytes (NHOKs). Knockdown of NRSF/REST by siRNA reduced cell viability only in $\mathrm{KB}$ cells in a time-dependent manner, and this effect was due to the activation of apoptosis components and DNA fragmentation. In addition, knockdown of NRSF/REST disrupted the mTOR signaling pathway which is a key survival factor in many types of cancer cells. For example, the phosphorylation of elF4G, elF4E and 4E-BP1 was significantly reduced in the KB cells upon NRSF/REST knockdown. These results imply that NRSF/REST plays an important role in the survival of oral cancer cells by regulating the mTOR signaling pathway.
\end{abstract}

\section{Introduction}

Neuron-restrictive silencer factor (NRSF), also called repressor element 1 (RE-1) silencing transcription factor (REST), is a major transcriptional repressor for neuron-specific genes in non-neuronal and neuronal progenitor cells (1-3) through

Correspondence to: Professor Chun Sung Kim, Department of Oral Biochemistry, College of Dentistry, Chosun University, 375 Seosukdong, Dong-gu, Gwangju 501-759, Republic of Korea

E-mail: cskim2@chosun.ac.kr

${ }^{*}$ Contributed equally

Key words: NRSF/REST, human oral squamous cell carcinoma, mTOR signaling, apoptosis histone deacetylation, chromatin remodeling, methylation and other mechanisms (4-6). NRSF/REST is a $116-\mathrm{kDa}$ protein that contains a DNA-binding domain with 8 zinc fingers and 2 repressor domains: one at the amino-terminus and the other at the carboxy-terminus of the protein $(1,7,8)$. NRSF/REST binds to a 21-bp consensus sequence of the neural restrictive silencer element (NRSE), also known as the RE-1, in the regulatory regions of many neuron-specific genes: ion channels, neurotransmitter and neurotropic receptors, neuronal cell adhesion molecules, neuronal growth factors, neuronal cytoskeleton, and others $(5,9)$. NRSF/REST mediates transcription repression through the association of its amino terminal repression domain with the $\mathrm{mSin} 3 /$ histone deacethylase $1 / 2$ (HDAC 1/2) complex (10-12) and through the association of its carboxy terminal repression domain with the CoREST/HDAC complex $(13,14)$.

Although NRSF/REST was first identified as a transcriptional repressor of neuronal genes in non-neuronal cells, recent studies have revealed seemingly paradoxical roles in tumor suppression and cancer progression.

NRSF/REST has a tumor-suppressor function in colorectal cancer (15). In RNAi-based genetic screening for tumor-suppressor genes in human mammary epithelial cells, NRSF/REST was 1 of 5 candidate genes that survived validation. Anchorage-independent growth was facilitated by knockdown of NRSF/REST in these cells, but suppressed by overexpression of NRSF/REST in a colon cancer cell line. In support of this view, NRSF/REST was mutated in several primary tumor specimen and colon cancer cell lines. The expression of a mutated form, a dominant-negative form of NRSF/REST, caused a stimulated PI(3)K pathway and subsequent transformation.

In contrast, NRSF/REST has an oncogenic function in medulloblastoma. Human medulloblastoma cell lines and many human medulloblastomas overexpress NRSF/REST compared with neuronal cells or fully differentiated neurons (16-18). NRSF/REST-expressing human medulloblastoma cells did not express NRSF/REST target genes that are important in tumorigenesis. In support of this view, REST-VP16, a recombinant transcription factor, by replacing the repressor domains of NRSF/REST with the activation domain of the herpes simplex virus protein VP16, operates through RE1/NRSE, 
competes with endogenous NRSF/REST for DNA binding and activates NRSF/REST target genes. As a result, the expression of REST-VP16 in these cells was shown to cause blockade of tumorigenicity (18-20).

In addition, NRSF/REST plays an oncogenic role in several neuroblastoma cells. Overexpression of NRSF in neuroblastoma cells was accompanied with repression of neuronal differentiation genes. Furthermore, NRSF/REST expression decreased and neuronal markers increased during differentiation-induction in these cells. Therefore, the overexpression of NRSF in neuronal cells blocks these cells from neuronal differentiation and produces the cancerous phenotype $(21,22)$.

Oral cancer, also called oral cavity cancer, is squamous cell carcinoma (SCC) that originates from squamous epithelium of the oral tongue and floor of the mouth (23), and is one of the top most commonly diagnosed cancer types in the world (24). Oral SCC represents $1 \sim 2 \%$ of all human malignancies, whereas survival rates are among the lowest of major cancers. It is characterized by a high degree of local invasiveness and a high rate of metastasis to cervical lymph nodes. The migration of oral SCC into maxillary and mandibular bones is a common clinical problem. Current treatment, including surgical removal of the lesion, is inadequate due to the wide exposure of carcinogens over a large mucosal surface. Hence, there is an unmet medical need to develop better diagnostic measures and therapeutic targets.

In the present study, we studied the roles of NRSF/NRST in regard to cell survival, apoptosis, and translational regulation in an oral cancer cell line. The results demonstrated that NRSF/REST was directly associated with the survival of oral cancer cells through the regulation of the mTOR signaling pathway.

\section{Materials and methods}

Cell culture. Normal human oral keratinocytes (NHOKs) were purchesed from the ScienCell Research Laboratories (Carlsbad, CA, USA). NHOKs were maintained in KGM and a supplementary growth factor bullet kit (Clonetics Corp., San Diego, CA, USA). Human oral squamous carcinoma KB cells were grown in MEM medium (Gibco, Grand Island, NY, USA) supplemented with $10 \%$ heat-inactivated fetal bovine serum, $100 \mathrm{U} / \mathrm{ml}$ penicillin/100 $\mu \mathrm{g} / \mathrm{ml}$ streptomycin (Gibco) and $0.1 \mu \mathrm{M}$ MEM non-essential amino acids (Gibco) at $37^{\circ} \mathrm{C}$ in a humidified atmosphere of $5 \% \mathrm{CO}_{2}$.

siRNAs and transfection. The day before transfection, $\mathrm{KB}$ cells or NHOKs were plated in 6-well plates at a density of $7 \times 10^{5}$ cells in each well. After overnight incubation, cells were transfected with NRSF siRNA using Lipofectamine 2000 transfection reagent (Invitrogen Life Technologies, Carlsbad, CA, USA) according to the manufacturer's instructions. Briefly, NRSF siRNA and Lipofectamine 2000 were each diluted in $50 \mu \mathrm{l}$ Opti-MEM I and incubated for $5 \mathrm{~min}$ at room temperature. The diluted NRSF siRNA and Lipofectamine 2000 were combined and then incubated for $20 \mathrm{~min}$ at room temperature. After incubation, NRSF siRNA/Lipofectamine 2000 complexes were added to each well. The cells were incubated at $37^{\circ} \mathrm{C}$ in a humidified atmosphere of $5 \% \mathrm{CO}_{2}$. NRSF siRNA and negative control siRNA were purchased from Bioneer
(AccuTarget ${ }^{\mathrm{TM}}$ Human genome-wide predesigned siRNA; no. 1128323, AccuTarget ${ }^{\mathrm{TM}}$ negative control siRNA; SN-1002, Daejeon, Korea). The concentrations of siRNAs were optimized to $50 \mathrm{pmol} /$ well. After transfection, on days 1, 2, 3 and 4 , the cells were re-transfected with the same siRNA for thorough knockdown of NRSF. siRNA-transfected cells were harvested at each days. Total proteins were prepared using RIPA buffer.

MTT assay. The day before transfection, $\mathrm{KB}$ cells and NHOKs were plated in 24-well plates at a density of $1.5 \times 10^{5}$ cells in each well. After overnight incubation, cells were transfected with NRSF siRNA using Lipofectamine 2000 transfection reagent according to the manufacturer's instructions. On days 1, 2, 3 and 4 , cells were re-transfected with the same siRNA. Fifty microliters of the $5 \mu \mathrm{g} / \mathrm{ml}$ stock solution of 3-(4,5-dimetylthiazol-2-yl)-2,5-diphenyltetrazolium bromide (Sigma-Aldrich, St. Louis, MO, USA) was added to each well, and the cells were incubated at $37^{\circ} \mathrm{C}$ in a humidified atmosphere of $5 \% \mathrm{CO}_{2}$ for $4 \mathrm{~h}$. After removal of the supernatant, $0.5 \mu \mathrm{l}$ of isopropanol was added to each well. Plates were incubated at room temperature on a horizontal shaker for $20 \mathrm{~min}$, and the optical density was measured in absorbance at $562 \mathrm{~nm}$ with a microplate reader (EL311SX; BIO TEX Instruments Inc., Cortland, NY, USA). Independent experiments were performed in triplicate and the Student's t-test was used for statistical analysis.

Western blot analysis. KB cells were plated in 6-well plates at a density of $7 \times 10^{5}$ cells/well. After overnight incubation, the cells were transfected with NRSF siRNA using Lipofectamine 2000 transfection reagent according to the manufacturer's instructions. On days 1, 2, 3 and 4, the cells were re-transfected with the same siRNA. siRNA-transfected cells were harvested on each day. For harvesting, the cells were washed twice with PBS. Cells were lysed in RIPA buffer [50 $\mu \mathrm{M}$ Tris- $\mathrm{HCl} \mathrm{pH}$ $7.5,150 \mu \mathrm{M} \mathrm{NaCl}, 0.25 \%$ sodium deoxycholate, $0.1 \%$ Nonidet P-40, 0.1\% SDS, $50 \mu \mathrm{M} \mathrm{NaF}, 0.5 \mu \mathrm{M}$ phenylmethylsulfonyl fluoride, $10 \mu \mathrm{M} \mathrm{Na}{ }_{3} \mathrm{VO}_{4}$ and Complete Mini protease inhibitor cocktail (Roche, Mannheim, Germany)] for $30 \mathrm{~min}$ on ice. The supernatant was transferred to a new tube after centrifugation at $15,000 \times \mathrm{g}$ for $15 \mathrm{~min}$ at $4^{\circ} \mathrm{C}$ (Sorvall centrifuge, Bad Homburg, Germany). The protein concentration was quantified by the BCA protein assay (Pierce, Rockford, IL, USA) using BSA as a standard. Approximately $50 \mu \mathrm{g}$ of protein from each lysate was solubilized in Laemmli sample buffer and then loaded onto 3-8 or 4-20\% gradient gel (Invitrogen Life Technologies). The protein was separated by electrophoresis at $120 \mathrm{~V}$ for $90 \mathrm{~min}$. The separated proteins were transferred to a polyvinylidene difluride nanofiber membrane (Amomedi, Gwangju, Korea). The membranes were blocked for $1 \mathrm{~h}$ with $5 \%$ milk at room temperature, followed by incubation overnight with primary antibodies against each of the following: anti-NRSF (07-579; Upstate Biotechnology, Billerica, MA, USA), anti-phospho-eIF4G (\#2441), anti-cleaved caspase-9 (\#9501), anti-cleaved caspase-7 (\#9491) (all from Cell Signaling Technology, Danvers, MA, USA), anti- $\beta$-actin (sc-47778, Santa Cruz Biotechnology, Santa Cruz, CA, USA). After washing 3 times with TBST (0.1\% Tween-20, $50 \mu \mathrm{M}$ Tris- $\mathrm{HCl} \mathrm{pH} 7.5$, $150 \mu \mathrm{M} \mathrm{NaCl}$ ), the membranes were incubated for $2 \mathrm{~h}$ with secondary antibodies and then washed 3 times with TBST. 
A

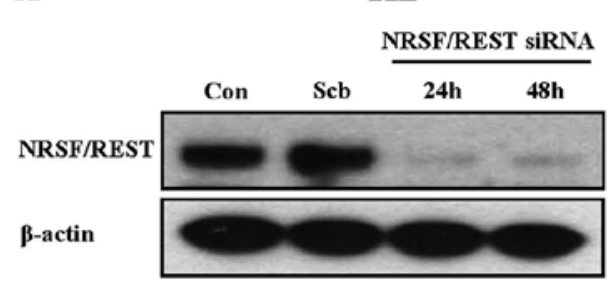

B

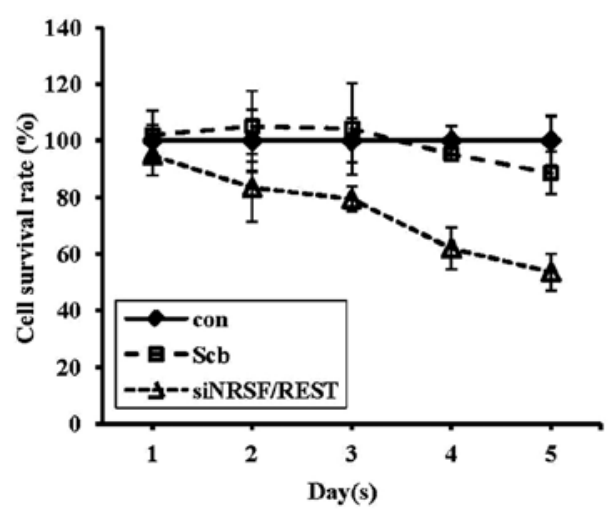

NHOK

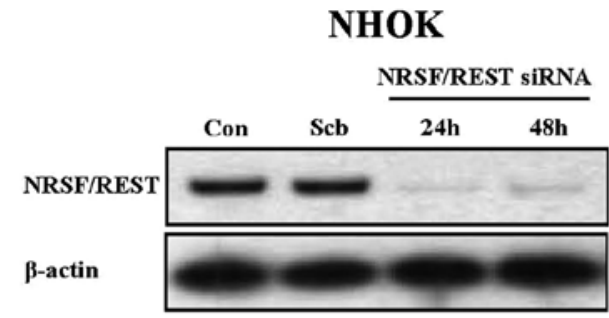

NHOK

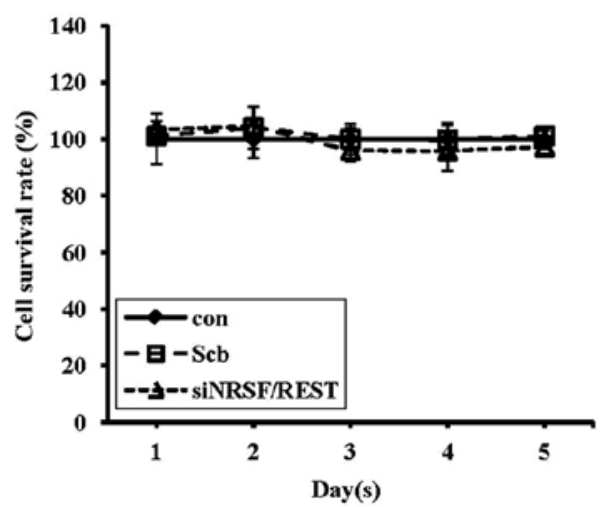

Figure 1. Effects of siRNA-mediated knockdown of NRSF/REST on the cell viability in KB cells and NHOKs. KB cells and NHOKs were transfected with NRSF/REST siRNA and non-specific siRNA ( $\mathrm{Scb}$ ), and whole protein was prepared every $24 \mathrm{~h}$ for 2 days post-transfection. (A) A reduction in the NRSF/ REST protein level by siRNA-mediated knockdown was detected by western blot analysis with the anti-NRSF/REST antibody. (B) Cell viability was assessed by the MTT assay every $24 \mathrm{~h}$ for 5 days. Data are shown as mean \pm SD from 3 independent experiments. NRSF/REST, neuron-restrictive silencer factor/ repressor element 1-silencing transcription factor; NHOK, normal human oral keratinocytes.

The membranes were detected using the WestSave Up ECL kit (ABFrontier, Seoul, Korea) and exposed to Kodak BioMax Light Film (Carestream Health Inc., Rochester, NY, USA).

DNA fragmentation assay. KB cells were plated in 6-well plates at a density of $7 \times 10^{5}$ cells in each well. Transfection of siRNAs was also performed as described previously. Cells were washed twice with PBS and pelleted by centrifugation at $400 \mathrm{x} \mathrm{g}$ for $3 \mathrm{~min}$. Cell pellets were lysed in lysis buffer (10 $\mu \mathrm{M}$ Tris-Cl, $\mathrm{pH} 7.5,10 \mu \mathrm{M}$ EDTA, $0.5 \%$ Triton $\mathrm{X}-100)$ and kept on ice for $30 \mathrm{~min}$. RNase A was added to a final concentration of $0.5 \mu \mathrm{g} / \mathrm{ml}$ and incubated for $1 \mathrm{~h}$ at $37^{\circ} \mathrm{C}$, followed by the addition of proteinase $\mathrm{K}$ to a final concentration of $0.2 \mu \mathrm{g} / \mathrm{ml}$ and incubated for $8 \mathrm{~h}$ at $50^{\circ} \mathrm{C}$. DNA was extracted using phenol:chloroform:isoamyl alcohol (25:24:1). DNA was precipitated with an equal volume of isopropanol, stored at $-70^{\circ} \mathrm{C}$ for $24 \mathrm{~h}$, and centrifuged at $15,000 \times \mathrm{g}$ for $15 \mathrm{~min}$ at $4^{\circ} \mathrm{C}$. The precipitated DNA was air-dried, resuspended in $30 \mu 1$ TE buffer and quantified by absorbance at $260 \mathrm{~nm}$ in a UV spectrophotometer (BioPhotometer, Eppendorf, Hamburg, Germany). Ten micrograms of DNA was applied to $1.5 \%$ agarose gel and electrophoresed at $50 \mathrm{~V}$ for $50 \mathrm{~min}$. DNA fragments were visualized using a UV transilluminator after ethidium bromide staining.

Statistical analysis. The data are reported as the mean \pm SD of 3 individual experiments performed in triplicate and presented as the mean. Statistical analysis was performed by a Student's t-test, and a P-value <0.05 was considered significant.

\section{Results}

Silencing of NRSF/REST expression decreases oral cancer cell viability. First, to verify whether NRSF/REST is expressed in KB cells and NHOKs, western blot analysis was performed. The data showed that KB carcinoma cells expressed NRSF/REST at a higher level than that in the NHOKs (Fig. 1A). To investigate the role of NRSF/REST in the cell viability of KB cells and NHOKs, we used siRNA to knockdown endogenous NRSF/REST. Specific suppression of NRSF/REST was assayed by western blot analysis. The expression level of NRSF/REST protein was significantly reduced in the NRSF/REST siRNA-transfected group compared to both the non-transfected and negative siRNA-transfected group. The data showed that NRSF/REST siRNA was successful in the knockdown of NRSF/REST protein expression (Fig. 1A).

Consequently, cell viability of these cells after NRSF/REST siRNA transfection was measured by MTT assay. Cell viability was significantly decreased up to $50 \%$ by NRSF/REST siRNA in the KB cells, whereas there was no effect of NRSF/REST siRNA on the cell viability of the NHOKs (Fig. 1B). This result indicates that NRSF/REST is important for KB cancer cell survival but is not for the normal oral cell line.

Downregulation of cell viability by NRSF/REST knockdown is due to apoptosis. To address whether the mechanism of the cytotoxic effect of NRSF/REST knockdown on the KB cells was related to apoptosis, the expression of apoptotic marker genes was determined by western blot analysis, and a DNA 
A

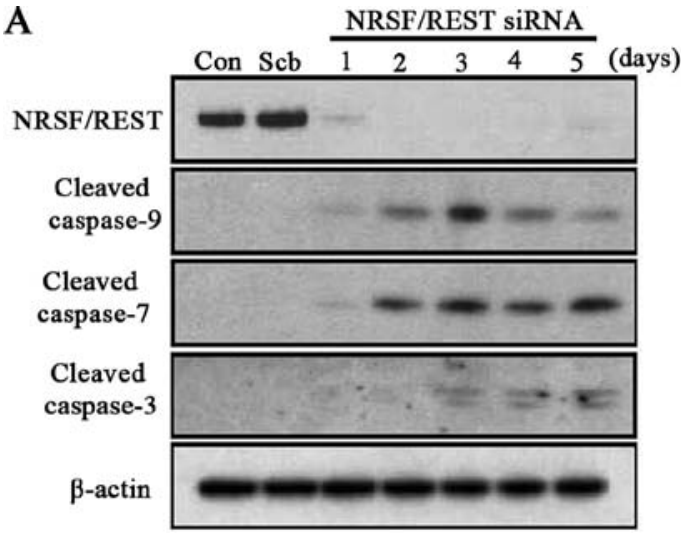

B

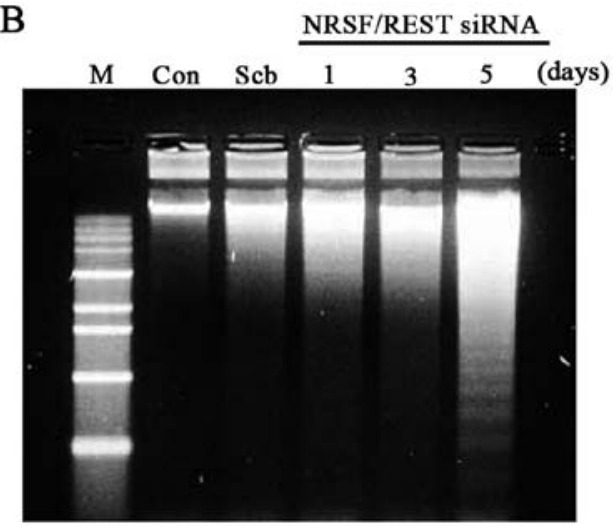

Figure 2. Apoptosis by knockdown of NRSF/REST in KB cells. KB cells were transfected with NRSF/REST siRNA and non-specific siRNA (Scb) over a time course of 5 days. (A) Apoptosis was assessed by western blot analysis using rabbit polyclonal NRSF/REST antibody and antibodies against cleaved caspase-3, -7 and -9. (B) DNA was prepared from NRSF/REST siRNA-transfected or non-specific siRNA-transfected cells and analyzed on a 1.5\% agarose gel. NRSF/REST, neuron-restrictive silencer factor/repressor element 1-silencing transcription factor.

A

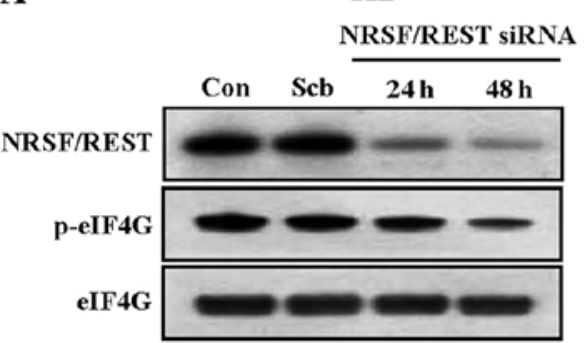

B

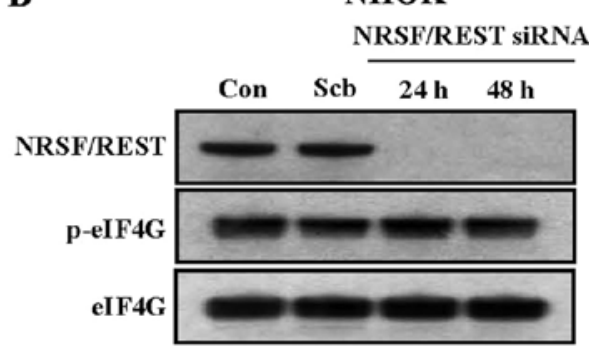

Figure 3. Inhibition of eIF4G phosphorylation. (A) KB cells and (B) NHOKs were transfected with NRSF/REST siRNA and non-specific siRNA (Scb), and whole cell protein was prepared every $24 \mathrm{~h}$ for 2 days post-transfection. The effect of NRSF/REST knockdown on eIF4G phosphorylation was assessed by western blot analysis using rabbit polyclonal NRSF/REST antibody and antibodies against phosphorylated and non-phosphorylated eIF4G. NRSF/REST, neuron-restrictive silencer factor/repressor element 1-silencing transcription factor; NHOK, normal human oral keratinocytes.

fragmentation assay was performed. The expression of cleaved caspase- 9 and -7 , activated forms of caspase- 9 and -7 , respectively, were significantly increased in the KB cells 2 days after transfection with NRSF/REST siRNA (Fig. 2A). In addition, caspase-3, the activator of caspase-activated DNase (CAD) or DNA fragmentation factor 40 (DFF40), was induced from day 3 following transfection with NRSF/REST siRNA (Fig. 2A) and this was correlated to the appearance of fragmented DNA (Fig. 2B) which is one of the major hallmarks of apoptotic cells.

$N R S F / R E S T$ regulates the phosphorylation of eIF4G. A recent study demonstrated that knockdown of NRSF/REST induced a decrease in eIF4G phosphorylation, a key downstream component of mTOR, in human neuroblastoma cells (25). Since the mTOR signaling pathway has a central role in the regulation of cancer cell growth by control of the initiation of mRNA translation into protein, we investigated the effect of NRSF/REST knockdown on the level of elF4G phosphorylation in both the KB cells and NHOKs. As shown in Fig. 3, western blot analysis indicated that the level of phosphorylated elF4G was significantly reduced in the NRSF/REST siRNA-treated KB cells but not in the NHOKs while the expression level of total eIF4G was not changed by the NRSF/REST siRNA transfection in both cell types.
NRSF/REST regulates the mTOR signaling pathway. eIF4G is one of the fundamental downstream targets of the mTOR signaling pathway (26). To confirm that the downregulation of eIF4G phosphorylation by knockdown of NRSF/REST is associated with the phosphorylation of mTOR, western blot analysis was performed. The phosphorylation of mTOR was significantly decreased following knockdown of NRSF/REST in the KB cells whereas the phosphorylation of mTOR after knockdown of NRSF/REST was slightly increased in the NHOKs (Fig. 4A). However, the endogenous levels of total mTOR were not affected by NRSF/REST siRNA in these cells (Fig. 4A).

In addition to elF4G, 4E-BP1 and elF4E are downstream components of the mTOR signaling pathway that directly activate the caspase-dependent translation process (27). To determine whether NRSF/REST knockdown affects these 2 downstream components of the mTOR signaling pathway, the phosphorylation of 4E-BP1 and eIF4E was assayed by western blot analysis. The phosphorylation of 4E-BP1 and eIF4E was significantly reduced in the KB cells with knockdown of the NRSF/REST compared to the negative siRNA-transfected cells. However, the endogenous levels of 4E-BP1 and eIF4E were not changed by NRSF/REST siRNA transfection (Fig. 4B). All together, these results indicate that NRSF/REST controls the mTOR signaling pathway for oral cancer cell survival. 

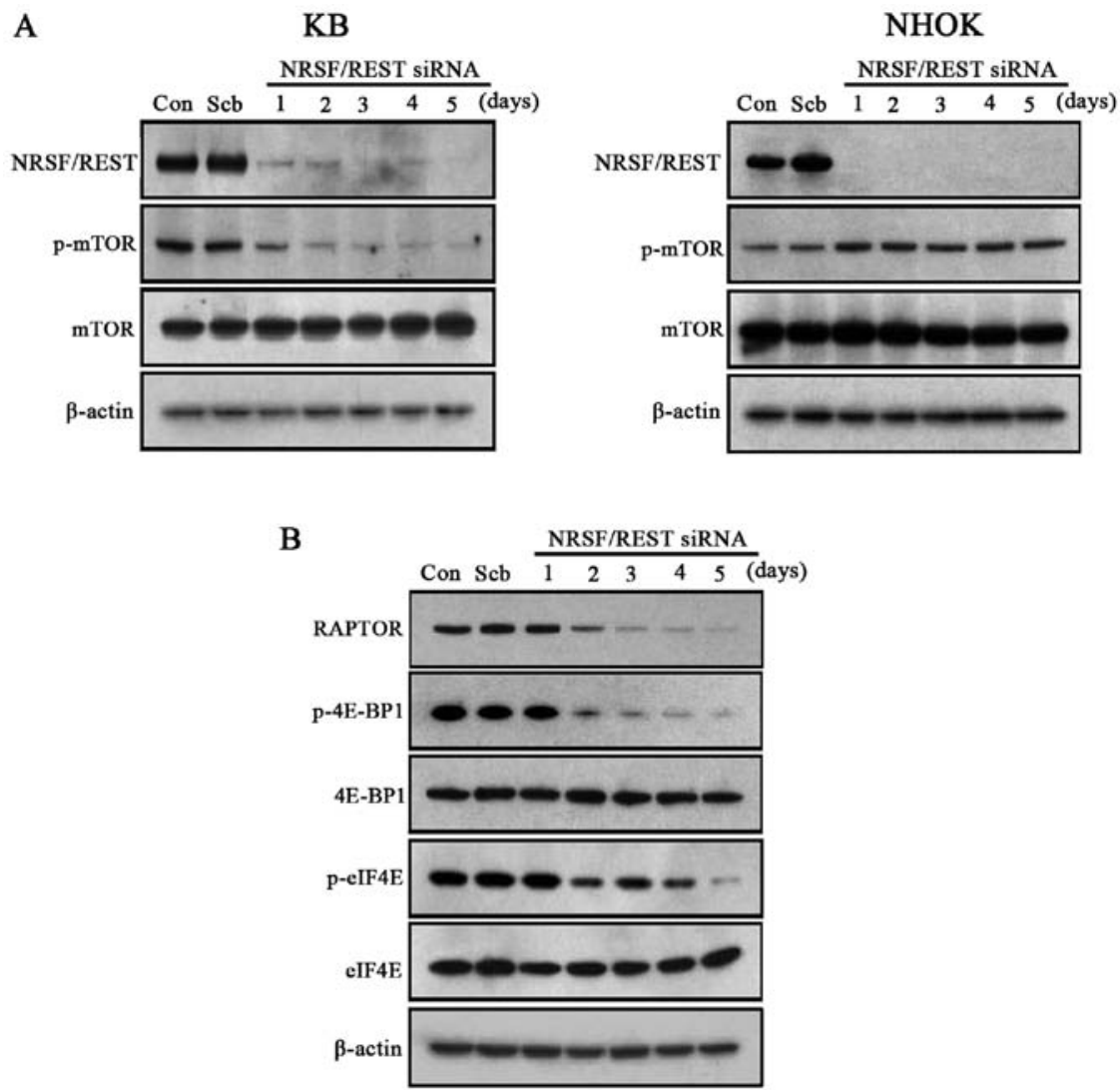

Figure 4. NRSF/REST regulates the phosphorylation of mTOR signaling pathway components. KB cells and NHOKs were transfected with NRSF/REST siRNA and non-specific siRNA (Scb) over a time course of 5 days, and whole lysates were prepared every 24 h for western blot analysis. (A) Inhibition of mTOR phosphorylation by NRSF/REST knockdown in KB cells and NHOKs. (B) Effect of NRSF/REST knockdown on the phosphorylation of downstream targets of the mTOR signaling pathway in KB cells. Phosphorylation of target proteins was assessed by western blot analysis using rabbit polyclonal NRSF/REST antibody and antibodies against phosphorylated and non-phosphorylated mTOR, 4E-BP1 and eIF4E. NRSF/REST, neuron-restrictive silencer factor/repressor element 1-silencing transcription factor; NHOK, normal human oral keratinocytes.

\section{Discussion}

NRSF/REST is a transcription factor that plays a major role in the repression of neuron-specific genes in non-neuronal cells via binding to the neuron restrictive silencer element (1-3) and is associated with various types of cancers $(15,16,28-31)$. It has been known to confer both tumorigenic and tumorsuppressor effects, depending on the cell type (20). Our results showed that KB oral cancer cells overexpressed NRSF/REST when compared with the normal oral keratinocytes, and siRNA-mediated knockdown of NRSF/REST significantly decreased KB cell viability. The activation of caspase components and fragmentation of DNA indicated that apoptosis was the mechanism of the observed KB cell death. Thus, we suggest that NRSF/REST may have an oncogenic function in $\mathrm{KB}$ oral cancer cells.

Although the association of NRSF/REST in various cancer types has been reported, the mechanism of action of NRSF/REST in cancer progression and tumorigenesis remains unknown. Recently, our laboratory studied the role of NRSF/REST in post-transcriptional regulation and found that it enhanced the phosphorylation of the eukaryotic translation initiation factor, elF4G (25). Since elF4G is one of the downstream components in the mTOR signaling pathway and mTOR is known to be a key survival factor in many cancer cells $(32,33)$, we reasoned that NRSF/REST may exert its oncogenic activity via the mTOR signaling pathway in KB oral cancer cells. As expected, siRNA-mediated NRSF/REST knockdown reduced the phosphorylation of elF4G and mTOR, and significantly inhibited the activation of other downstream components (elF4G, elF4E, and 4E-BP1) (Fig. 3 and 4) strongly suggesting that NRSF/REST controls the mTOR pathway in $\mathrm{KB}$ oral cancer cells.

It is important to point out that the effects of NRSF/REST knockdown described in the present study are reminiscent of that of rapamycin in MDA-MB 231 breast cancer cells (34). This study reported that a high dose of rapamycin induced apoptosis in MDA-MB231 cells and it was correlated with suppression of the phosphorylation of the mTOR substrate, 4E-BP1. Furthermore, the study provided evidence that complete dissociation of Raptor from the mTOR complex is necessary for rapamycin-mediated apoptosis in MDA-MB-231 cells. Therefore, it is tempting to speculate that NRSF/REST knockdown in KB oral cancer cells may disrupt the formation of the active mTOR complex by dysregulating components of the mTOR complex such as Raptor.

Notably, NRSF/REST knockdown did not cause cell death and mTOR regulation in NHOKs while they expressed detectable amount of NRSF/REST although much lower than that in $\mathrm{KB}$ cancer cells. It has been shown that, when overexpressed, NRSF/REST can be localized in both the nucleus and cytoplasm; cytoplasmic NRSF/REST can upregulate transla- 
tion and enhance elF4G phosphorylation in neuroblastoma cells (25). Thus, aberrant high expression of NRSF/REST in $\mathrm{KB}$ oral cancer cells compared to normal NHOKs may explain the role of NRSF/REST in cell survival by controlling the mTOR pathway only in KB cells.

In summary, we demonstrated that NRSF/REST functions as a regulator of the mTOR signaling pathway in KB oral cancer cells and is important for cancer cell survival. Further delineation of the mechanism of NRSF/REST action in the mTOR signaling pathway may provide insight into the development of a novel oral cancer treatment.

\section{Acknowledgements}

The present study was supported by a grant from the National Research Foundation of Korea (NRF) funded by the Korea government MSIP (no. 2008-0062283)

\section{References}

1. Chong JA, Tapia-Ramírez J, Kim S, Toledo-Aral JJ, Zheng Y, Boutros MC, Altshuller YM, Frohman MA, Kraner SD and Mandel G: REST: a mammalian silencer protein that restricts sodium channel gene expression to neurons. Cell 80: 949-957, 1995.

2. Schoenherr CJ and Anderson DJ: The neuron-restrictive silencer factor (NRSF): a coordinate repressor of multiple neuron-specific genes. Science 267: 1360-1363, 1995.

3. Jones FS and Meech R: Knockout of REST/NRSF shows that the protein is a potent repressor of neuronally expressed genes in non-neural tissues. Bioessays 21: 372-376, 1999.

4. Palm K, Belluardo N, Metsis M and Timmusk T: Neuronal expression of zinc finger transcription factor REST/NRSF/XBR gene. J Neurosci 18: 1280-1296, 1998.

5. Lunyak VV, Burgess R, Prefontaine GG, Nelson C, Sze SH, Chenoweth J, Schwartz P, Pevzner PA, Glass C, Mandel G and Rosenfeld MG: Corepressor-dependent silencing of chromosomal regions encoding neuronal genes. Science 298: 1747-1752, 2002.

6. Ballas $\mathrm{N}$ and Mandel G: The many faces of REST oversee epigenetic programming of neuronal genes. Curr Opin Neurobiol 15: 500-506, 2005.

7. Tapia-Ramírez J, Eggen BJ, Peral-Rubio MJ, Toledo-Aral JJ and Mandel G: A single zinc finger motif in the silencing factor REST represses the neural-specific type II sodium channel promoter. Proc Natl Acad Sci USA 94: 1177-1182, 1997.

8. Thiel G, Lietz M and Cramer M: Biological activity and modular structure of RE-1-silencing transcription factor (REST), a repressor of neuronal genes. J Biol Chem 273: 26891-26899, 1998.

9. Bruce AW, Donaldson IJ, Wood IC, Yerbury SA, Sadowski MI, Chapman M, Göttgens B and Buckley NJ: Genome-wide analysis of repressor element 1 silencing transcription factor/neuronrestrictive silencing factor (REST/NRSF) target genes. Proc Natl Acad Sci USA 101: 10458-10463, 2004.

10. Naruse Y, Aoki T, Kojima T and Mori N: Neural restrictive silencer factor recruits $\mathrm{mSin} 3$ and histone deacetylase complex to repress neuron-specific target genes. Proc Natl Acad Sci USA 96: 13691-13696, 1999.

11. Grimes JA, Nielsen SJ, Battaglioli E, Miska EA, Speh JC, Berry DL, Atouf F, Holdener BC, Mandel G and Kouzarides T: The co-repressor $\mathrm{mSin} 3 \mathrm{~A}$ is a functional component of the REST-CoREST repressor complex. J Biol Chem 275: 9461-9467, 2000.

12. Roopra A, Sharling L, Wood IC, Briggs T, Bachfischer U, Paquette AJ and Buckley NJ: Transcriptional repression by neuron-restrictive silencer factor is mediated via the Sin3-histone deacetylase complex. Mol Cell Biol 20: 2147-2157, 2000.

13. Ballas N, Battaglioli E, Atouf F, Andres ME, Chenoweth J, Anderson ME, Burger C, Moniwa M, Davie JR, Bowers WJ, Federoff HJ, Rose DW, Rosenfeld MG, Brehm P and Mandel G: Regulation of neuronal traits by a novel transcriptional complex. Neuron 31: 353-365, 2001.
14. You A, Tong JK, Grozinger CM and Schreiber SL: CoREST is an integral component of the CoREST-human histone deacetylase complex. Proc Natl Acad Sci USA 98: 1454-1458, 2001.

15. Westbrook TF, Martin ES, Schlabach MR, Leng Y, Liang AC, Feng B, Zhao JJ, Roberts TM, Mandel G, Hannon GJ, Depinho RA, Chin L and Elledge SJ: A genetic screen for candidate tumor suppressors identifies REST. Cell 121: 837-848, 2005.

16. Lawinger P, Venugopal R, Guo ZS, Immaneni A, Sengupta D, Lu W, Rastelli L, Marin Dias Carneiro A, Levin V, Fuller GN, Echelard Y and Majumder S: The neuronal repressor REST/ NRSF is an essential regulator in medulloblastoma cells. Nat Med 6: 826-831, 2000.

17. Su X, Kameoka S, Lentz S and Majumder S: Activation of REST/NRSF target genes in neural stem cells is sufficient to cause neuronal differentiation. Mol Cell Biol 24: 8018-8025, 2004.

18. Fuller GN, Su X, Price RE, Cohen ZR, Lang FF, Sawaya R and Majumder S: Many human medulloblastoma tumors overexpress repressor element-1 silencing transcription (REST)/neuron-restrictive silencer factor, which can be functionally countered by REST-VP16. Mol Cancer Ther 4: 343-349, 2005.

19. Immaneni A, Lawinger P, Zhao Z, Lu W, Rastelli L, Morris JH and Majumder S: REST-VP16 activates multiple neuronal differentiation genes in human NT2 cells. Nucleic Acids Res 28: 3403-3410, 2000.

20. Su X, Gopalakrishnan V, Stearns D, Aldape K, Lang FF, Fuller G, Snyder E, Eberhart CG and Majumder S: Abnormal expression of REST/NRSF and Myc in neural stem/progenitor cells causes cerebellar tumors by blocking neuronal differentiation. Mol Cell Biol 26: 1666-1678, 2006.

21. Nishimura E, Sasaki K, Maruyama K, Tsukada $\mathrm{T}$ and Yamaguchi K: Decrease in neuron-restrictive silencer factor (NRSF) mRNA levels during differentiation of cultured neuroblastoma cells. Neurosci Lett 211: 101-104, 1996.

22. Higashino K, Narita T, Taga T, Ohta S and Takeuchi Y: Malignant rhabdoid tumor shows a unique neural differentiation as distinct from neuroblastoma. Cancer Sci 94: 37-42, 2003.

23. Shi Z and Stack MS: Molecules of cell adhesion and extracellular matrix proteolysis in oral squamous cell carcinoma. Histol Histopathol 25: 917-932, 2010.

24. Parkin DM, Bray F, Ferlay J and Pisani P: Global cancer statistics, 2002. CA Cancer J Clin 55: 74-108, 2005.

25. Kim CS, Hwang CK, Song KY, Choi HS, Kim do K, Law PY, Wei LN and Loh HH: Novel function of neuron-restrictive silencer factor (NRSF) for posttranscriptional regulation. Biochim Biophys Acta 1783: 1835-1846, 2008.

26. Mamane Y,Petroulakis E,LeBacquerO and Sonenberg N: mTOR, translation initiation and cancer. Oncogene 25: 6416-6422, 2006.

27. Laplante M and Sabatini D: mTOR signaling in growth control and disease. Cell 149: 274-293, 2012.

28. Palm K, Metsis M and Timmusk T: Neuron-specific splicing of zinc finger transcription factor REST/NRSF/XBR is frequent in neuroblastomas and conserved in human, mouse and rat. Brain Res Mol Brain Res 72: 30-39, 1999.

29. Coulson JM, Edgson JL, Woll PJ and Quinn JP: A splice variant of the neuron-restrictive silencer factor repressor is expressed in small cell lung cancer: a potential role in derepression of neuroendocrine genes and a useful clinical marker. Cancer Res 60: 1840-1844, 2000.

30. Tawadros T, Martin D, Abderrahmani A, Leisinger HJ, Waeber G and Haefliger JA: IB1/JIP-1 controls JNK activation and increased during prostatic LNCaP cells neuroendocrine differentiation. Cell Signal 17: 929-939, 2005.

31. Lv H, Pan G, Zheng G, Wu X, Ren H, Liu Y and Wen J: Expression and functions of the repressor element 1 (RE-1)-silencing transcription factor (REST) in breast cancer. J Cell Biochem 110: 968-974, 2010.

32. Hay N and Sonenberg N: Upstream and downstream of mTOR. Genes Dev 18: 1926-1945, 2004.

33. Yuan R, Kay A, Berg WJ and Lebwohl D: Targeting tumorigenesis: development and use of mTOR inhibitors in cancer therapy. J Hematol Oncol 2: 45, 2009.

34. Yellen P, Saqcena M, Salloum D, Feng J, Preda A, Xu L, Rodrik-Outmezguine L and Foster DA: High-dose rapamycin induces apoptosis in human cancer cells by dissociating mTOR complex 1 and suppressing phosphorylation of 4E-BP1. Cell Cycle 22: 3948-3956, 2011. 